\section{Kongenitale Syphilis nimmt in den USA zu}

Eine aktuelle Analyse der in den USA gemeldeten Fälle zeigt einen deutlichen Anstieg der konnatalen Lues innerhalb der letzten Jahre. Dies ist umso bedenklicher, als das Screening auf Syphilis in den meisten Bundesstaaten gesetzlich vorgeschrieben ist.

E ine unbehandelte Lues, vor allem in ᄃder Frühschwangerschaft, kann zu Abort, neonatalem Tod oder schweren Schädigungen des Kindes führen. Diese Komplikationen lassen sich durch den frühzeitigen Infektionsnachweis bei der Mutter und durch antibiotische Behandlung verhindern (s. Übersicht Seite 348). Die Centers for Disease Control and Prevention (CDC) empfehlen ein serologisches Syphilis-Screening von Schwangeren im I. Trimenon oder bei der ersten durchgeführten Pränataldiagnostik. Seit 2003 ist ein Syphilis-Screening in 43 USBundesstaaten gesetzlich vorgeschrieben. In einer Publikation machten die CDC auf einen erneuten Anstieg der konna- talen Lues aufmerksam. Während die Inzidenz der kongenitalen Syphilis in den USA 1990 noch bei 17,3 Fällen pro 100.000 Lebendgeburten lag, sank sie bis 2004 auf 0,8/100.000. Ab 2005 zeigte sich jedoch ein erneuter Anstieg - 2008 betrug die Inzidenz bereits 10,1/100.000. Besonders häufig waren Afroamerikaner betroffen $(34,6 / 100.000)$.

Die Auswertung ergab, dass von 431 gemeldeten Fällen im Jahr 2008 bei 125 Müttern (29\%) keine pränatale Vorsorge durchgeführt worden war und die Syphilis somit erst bei der Entbindung diagnostiziert wurde. Von den 276 Fällen, bei denen eine Pränataldiagnostik erfolgte, wurden 75 Frauen erstmals innerhalb der letzten 30 Tage vor der Entbindung auf Syphilis untersucht. Bei 67 Frauen mit einer Pränataldiagnostik früher als 30 Tage vor der Entbindung erfolgte keine antibiotische Behandlung. $2008 \mathrm{kam}$ es in 6\% der Fälle zu einer Totgeburt, 1\% der infizierten Kinder starb innerhalb von 30 Tagen nach der Entbindung.

Kommentar: Es ist erstaunlich, dass in einem Land wie den USA eine sexuell übertragene Infektionskrankheit bei Schwangeren unerkannt bleibt und es dadurch zur kongenitalen Syphilis kommt. Diagnostik und Therapie der Lues sind seit vielen Jahren etabliert und sicher. Der erneute Anstieg der kongenitalen Syphilis verdeutlicht die Probleme des amerikanischen Gesundheitswesens.

Prof. Dr. Tino F. Schwarz

Centers for Disease Control and Prevention. Congenital syphilis - United States, 20032008. Morbidity and Mortality Weekly Report 2010; 59: 413-7. 